East African Medical Journal Vol. 86 No. 3 March 2009

AGE AT FIRST DOSE OF MEASLES VACCINATION IN ETHIOPIA

Y. Berhane, MD, PhD, Professor, Epidemiology and Public Health, Addis Continental Institute of Public Health, W. W. Schluter, MD, Medical Officer, WHO Ethiopia Country Office, P.O Box 26752-1000 Addis Ababa, Ethiopia, F. Oyewole, EPI Team Leader, WHO Ethiopia Country Office, currently EPI Focal Person, MD, WHO Ghana Country Office and O. A. Babaniyi, WHO Ethiopia Country Representative, P. O. Box 26751- 1000, Addis Ababa, Ethiopia

Request for reprints to: Prof. Y. Berhane, Addis Continental Institute of Public Health P.O. Box 26751- 1000, Addis Ababa, Ethiopia

\title{
AGE AT FIRST DOSE OF MEASLES VACCINATION IN ETHIOPIA
}

\author{
Y. BERHANE, W. W. SCHLUTER, F. OYEWOLE and O. A. BABANIYI
}

\begin{abstract}
Background: Although measles vaccination is recommended to be given at nine months of age in Ethiopia and in most of sub-Saharan Africa, no information is available about the age at which children actually receive their first dose of measles vaccine. This has important implications in terms of preventing infection and averting epidemics of measles.

Objective: To determine the age at which Ethiopian children actually receive their first dose of measles vaccine.

Design: Cross sectional study.

Setting: All major vaccination facilities including private and non-governmental health facilities that were registered with the Addis Ababa city Administration Health Bureau.

Subjects: A total of 17,674 records of children who received measles vaccination in health facilities were reviewed and in rural areas 615 children were surveyed over one year period September 2004 to August 2005.

Results: In both the urban and the rural settings the median age of children at first dose of measles vaccination was nine months. In the rural areas only $19.8 \%$ of children had vaccination cards. Measles coverage by card and history in rural areas was $84.4 \%$. Many children from the rural site received measles vaccination during supplemental immunisation activities (SIAs) rather than from routine vaccination programmes. Measles coverage significantly varies among sub-cities in Addis Ababa.

Conclusion: Vaccination practices and measles coverage levels do not support delaying the first measles vaccine dose. Strengthening the routine vaccination programmes must receive priority before changing recommended age for the first dose of measles vaccine.
\end{abstract}

\section{INTRODUCTION}

Vaccine preventable diseases are among the major causes of childhood morbidity and mortality in Ethiopia (1-3). Measles is the commonest in occurrence and the number of deaths from measles in Ethiopia contributes substantially to the worldwide measles mortality burden $(3,4)$. Measles can be fatal or cause permanent disability. For example, it is one of the important causes of preventable blindness among schoolaged children in developing countries $(2,5)$. A community based seroprevalencestudy indicated that most people in Ethiopia are infected with measles at a very young age (6). Thus the need for children receiving measles vaccine at the recommended age is not disputed.

The first measles dose is recommended to be given at the age of nine months. However child caregivers have not been fully complying with the prescribed schedule; and some children remain unvaccinated at the recommended time.
Several studies have shown that there are many factors that influence the compliance and acceptance of vaccination in Ethiopia $(3,7,8)$. The availability and the quality of services are among the major causes of non-compliance to schedule (3). The socio-economic status of the caregivers and health care utilisation culture in the residential context are also factors associated with vaccination schedule compliance (8). The success of the immunisation programme is dependent on achieving sustained high-level vaccination coverage in the routine activities thus it is imperative to study the utilisation of and compliance pattern with the routine vaccination programme (7-9).

A measles technical advisory group (TAG) was convened by WHO's Regional Office for Africa in 2005. This advisory group questioned the feasibility of shifting the age at first opportunity for measles vaccine from the current nine months of age to an older age (perhaps 12 months to 15 months) to maximise the benefit of 
higher seroconversion rates among older aged children (10). However, when considering the appropriate age at which to recommend measles vaccination, it became evident that there was insufficient information available pertaining to the age at which African children actually receive their first dose of measles vaccine. Thus this study was undertaken to determine the age at which children receive their first dose of measles vaccine in urban and rural African settings.

\section{MATERIALS AND METHODS}

This study utilised cross sectional study design to determine the age at which children receive their first dose of measles vaccine in urban and rural populations. It used two approaches to collect data; health facility record review in urban area and household survey in rural areas. The health facility-based cross sectional study was conducted in Addis Ababa. Vaccination records were examined in all major vaccination facilities including private and non-governmental health facilities that were registered with the Addis Ababa City Administration Health Bureau. Data were abstracted from vaccination register by trained nurses and health officers using a format developed for the purpose of this study. On average, two to three days were needed to collect the relevant data from the vaccination register in each health facility.

The age at first dose of measles vaccine in a rural setting was determined by conducting a cross-sectional household survey in rural communities covered by the Butajira health and demographic surveillance system. The site was chosen because of the availability of a birth registration system that was established in 1987 (11). All children aged 12 to 23 months were identified from the surveillance data base. Based on the list obtained from the database, house-tohouse visits were made to identify the children and obtain data pertaining to their vaccination status. A questionnaire was developed to collect relevant information for the purpose of the study. Data were collected by trained enumerators; training was provided for one day on how to locate respondents and collect relevant information using the questionnaire. The enumerators have had many years of experience in data collection at community level.

Data were processed using Epi Info statistical package version 6.04. The primary analysis was to calculate the proportion of children receiving measles vaccination at the recommended age, i.e., nine months. The median age at vaccination was also computed separately for rural and urban residents.

The study was ethically approved by the Scientific and Ethical Committee of the Faculty of Medicine, Addis Ababa University. All potential participants were informed about the purpose of the study and only those who consented to participate were included in the study. Children from the urban site at the time of the survey had already received their measles vaccination, thus there was no need for interventions about vaccination. However, for rural children who had not completed the primary vaccination series, their caregivers were advised to seek vaccination for their children at the nearest vaccination centre.

\section{RESULTS}

A total of 18,660 records of children who received vaccination at the vaccination centres in Addis Ababaduring theoneyearstudy period (September 2004 to August 2005) were reviewed. Of these, 986 $(5.3 \%)$ of records had no age recorded. Therefore the analysis was completed for the 17,674 children whose record contained age.

Overall, the median age at first measles vaccination was 9.3 months. As shown in Table 1, $72.2 \%$ of children received their vaccination at the age of nine months. Only 968 (5.5\%) of the children with recorded age received their first dose of measles vaccination earlier than the recommended age. Another 3,938 (22.3\%) of children received their first dose of measles vaccination at ten months of age or after. The age group distribution at which children received their first dose of measles vaccination was not significantly different by sex (Chi square test= $2.52 ; \mathrm{df}=4$; $\mathrm{p}$-value $=0.64$ ).

\section{Table 1}

Measles vaccination time by age and sex of children who received vaccination in health institutions in Addis

\begin{tabular}{lllllll}
\hline $\begin{array}{l}\text { Child age at vaccination } \\
\text { (in months) }\end{array}$ & \multicolumn{2}{c}{ Female } & \multicolumn{3}{c}{ Maba } \\
& No. & $(\%)$ & No. & $(\%)$ & No. & $(\%)$ \\
\hline$<9$ & 436 & 5.4 & 491 & 5.7 & 968 & 5.5 \\
9 & 5806 & 72.0 & 6275 & 72.6 & 12764 & 72.2 \\
$10-11$ & 1529 & 19.0 & 1564 & 18.1 & 3299 & 18.7 \\
$\geq 12$ & 295 & 3.6 & 319 & 3.7 & 639 & 3.60 \\
\hline
\end{tabular}


The percentage of children who received their first dose of measles vaccine at nine months of age was similar among all three categories of health facility ownership (government, private, and NGO). Overall, however, children who were taken to private and NGO health facilities were more likely to receive their first dose of measles vaccine before nine months of age (Chi square test $=174.81 ; \mathrm{df}=8 ; \mathrm{P}$-value $=0.0001)($ Table 2$)$.

\section{Table 2}

Measles vaccination time by age and sex of children who received vaccination in health institutions in Addis Ababa classified by ownership

\begin{tabular}{|c|c|c|c|c|c|c|}
\hline \multirow{2}{*}{$\begin{array}{l}\text { Child age at vaccination } \\
\text { (in months) }\end{array}$} & \multicolumn{2}{|c|}{ Government } & \multirow[b]{2}{*}{ No. } & \multirow{2}{*}{$\begin{array}{l}\text { Private } \\
(\%)\end{array}$} & \multicolumn{2}{|c|}{ NGO } \\
\hline & No. & $(\%)$ & & & No. & $(\%)$ \\
\hline $6.0-8.9$ & 729 & 4.9 & 139 & 8.2 & 70 & 9.8 \\
\hline $9.0-9.9$ & 10788 & 72.2 & 1251 & 73.7 & 501 & 70.1 \\
\hline $10.0-11.9$ & 2954 & 19.8 & 193 & 11.4 & 112 & 15.7 \\
\hline $12.0-14.9$ & 247 & 1.7 & 49 & 2.9 & 17 & 2.4 \\
\hline $15+$ & 230 & 1.5 & 65 & 3.8 & 15 & 2.1 \\
\hline Total & 14948 & 100 & 1697 & 100 & 715 & 100 \\
\hline
\end{tabular}

Figure 1

Percentage (and 95\% confidence interval) of children who receive measles vaccination at nine months in health institutions in Addis Ababa classified by sub-city

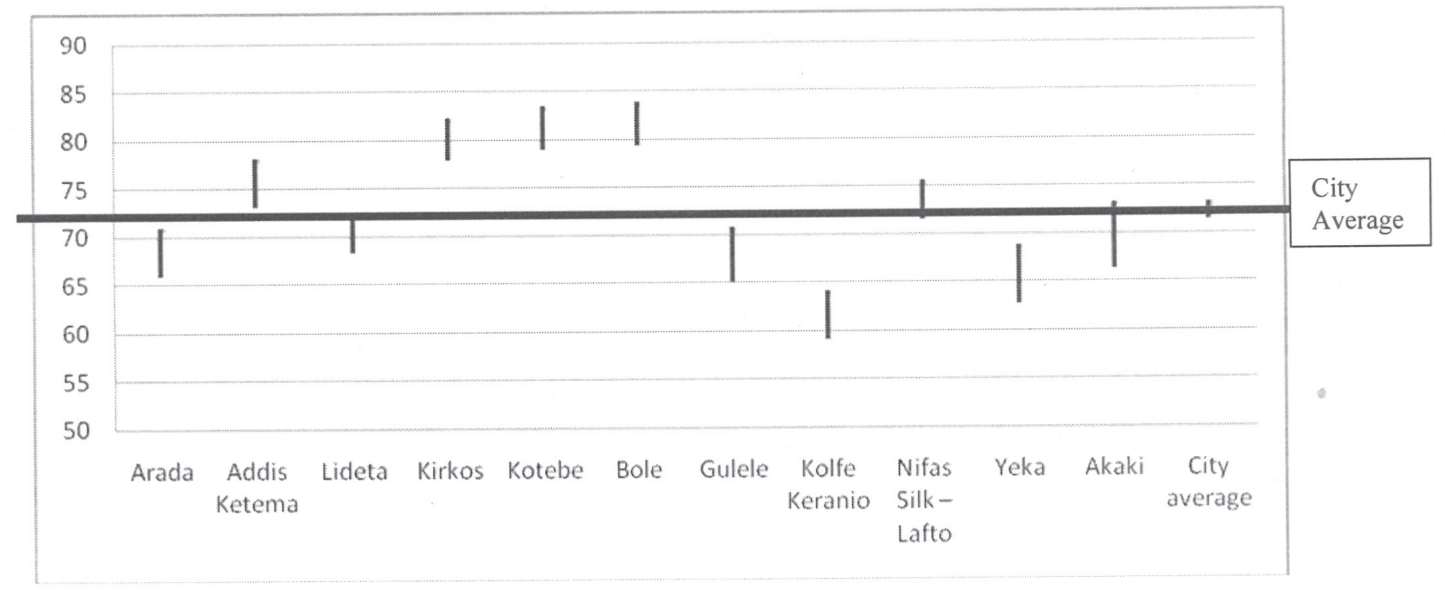

Table 3

Measles vaccination status of children in the age group 12-23 months in rural Meskan and Mareko district, SNNPR. 2005

\begin{tabular}{lcc}
\hline Measles vaccination status & Frequency & $(\%)$ \\
\hline Never vaccinated & 77 & 10.1 \\
Vaccinated and card seen & 122 & 19.8 \\
Vaccinated but card not seen (only history) & 397 & 64.6 \\
Status Unknown & 19 & 3.1 \\
\hline Total & 615 & 100 \\
\hline Measles coverage by card and history & $519 / 615$ & 84.4 \\
(Vaccinated for measles/ total children observed) & $122 / 615$ & 19.8 \\
$\begin{array}{l}\text { Measles coverage by card } \\
\text { (Vaccinated for measles at nine months/total children observed }\end{array}$ & & \\
\hline
\end{tabular}


As shown in Figure 1, there is no uniformity in the first measles dose coverage among sub-cities. Four of the sub-cities had significantly ( $\mathrm{P}$-value $<0.05$ ) lower first measles dose coverage compared to the city average.

In the rural site a total of 615 children in the age group of 12-23 months were identified for the purpose of this study. All, $84.4 \%$ of children had received at least one dose of measles vaccine by vaccination card or by caregivers' history. Only $19.8 \%$ of children had a vaccination card to verify their vaccination status. The median age at the time of first measles vaccination was nine months (range from 9-18 months) (Table 3). The majority of the children $(81.8 \%)$ were reportedly vaccinated at the age of nine months.

\section{DISCUSSION}

This study revealed that in Ethiopia the median age of first measles vaccination is nine months in both the urban and rural sites where the evaluation was completed. Additionally, it appears that most children actually receive their first dose of measles vaccination at the recommended age of nine months. The majority of children $(>97 \%)$ who actually received their first dose of measles vaccination did so before their first birthday. However; not all children received vaccination through the routine vaccination services in both urban and rural areas. In rural areas only $13 \%$ of all children had vaccination card to verify their vaccination status. In the urban study site, Addis Ababa, only the timing of the first measles vaccination was determined among those who came to the clinic, but the proportion of children vaccinated against measles was not determined. It should also be noted that caregiver reported age of children may not be accurate in a society where the use of calendar time is not common. Although the study tried to utilise local events to assist caregivers with recall to better estimate their children's age, this mechanism cannot guarantee reporting accuracy. Age heaping, which is a common phenomenon in many household surveys, could have been aggravated in this study due to the common reference made to nine months of age as the appropriate time to receive measles vaccination. As seen in other surveys the proportion of children with vaccination cards is much lower than the reported vaccination coverage (13).

Increasing availability of vaccination opportunities in closer proximity such as offering measles vaccination at outreach sites and during mass vaccination campaign programmes has greatly reduced the barriers for vaccination related to acceptance and quality of services that were mainly due to inaccessibility and inadequate awareness $(8,9)$. It is however, important to consider whether high coverage for measles vaccination can be sustained without mass vaccination campaigns.

Caregivers reported that their children receive measles vaccination mainly during mass vaccination campaigns $(14,15)$ indicating failure in access or utilisation of the routine vaccination programme. Strengthening the routine vaccination programme is essential in order to ensure sustainability of the gains in child health programmes. It is also important that some highly prevalent conditions such as HIVinfection or malnutrition can reduce maternally derived measles immunity and pose serious challenges to increasing the age at which the first dose of measles vaccination is given among children in sub-Saharan Africa. In some reports, measles has accounted for over $20 \%$ of all deaths among children less than five years of age in famine stricken populations in Ethiopia (16). As drought and famine are not infrequent in the continent, protecting children at an earlier age is a prudent measure. A study in Addis Ababa showed sero-negativity for measles antibodies decreases quickly from the age of nine months (6) indicating that most infants are vulnerable for measles infection before their first birthday. In a recent epidemic in South Africa, over $40 \%$ of the affected children were less than nine months of age (10).

Another important factor that needs consideration is the field efficacy of the measles vaccine for which very little is known in the sub-Saharan context. One study in Ethiopia has reported a low vaccine efficacy of $66 \%$ in actual field setting (17). If such is the field efficacy of the measles vaccine, giving the first dose at an early age and a booster dose at a later age is more appropriate.

In conclusion, this study indicates that not every child is vaccinated at the recommended nine months of age yet. Most of the children especially in rural areas are vaccinated during campaigns and not in routine services. Thus before considering to change the age of first measles dose, strengthening the routine immunisation programme to reach all eligible children must receive priority.

\section{ACKNOWLEDGEMENT}

To the WHO country office in Ethiopia in providing funds to the field work of the study. Special thanks for the health facilities and communities that participated in the study. 


\section{REFERENCES}

1. Mekasha, A., Lemma, F. and Shiferaw, T. Child health problems in Ethiopia. Ethiop. J. Health. Dev. 1995; 9:167-187.

2. Berhane, Y. and Yigzaw, A. Vaccine preventable diseases. In: Berhane, Y., Hailemariam, O. and Kloos (Eds): The epidemiology and ecology of health and disease in Ethiopia. Shama. Books. Addis. Ababa. Ethiopia. 2006; 354-371.

3. Berhane, Y., Bekele, A. and Tesfaye, F. Immunization (EPI) in Ethiopia: Acceptance, coverage, and sustainability. Ethiop. Med. J. 2000; 38 (Supp I) 1: $1-60$.

4. Stein, C.E., Birmingham, M., Kurian, M., et al. The global burden of measles in the year 2000a model that uses country-specific indicators. J. Infect. Dis. 2003; 187 (Suppl 1): S8-S14.

5. Kello, A. B. and Gilbert, C. Causes of severe visual impairment and blindness in children in schools for the blind in Ethiopia. Br. J. Ophthalmol. 2003; 87: 526-530.

6. Enquselassie, F., Ayele, W., Dejene, A. et al. Seroepidemiology of measles in Addis Ababa, Ethiopia: implications for control through vaccination. Epidemiol. Infect. 2003; 130: 507-519.

7. Berhane, Y. and Pickering, J. Are reminder stickers effective in reducing immunization dropout rates in Addis Ababa, Ethiopia? J. Trop. Med. Hyg. 1993; 96: 139-145.

8. Streefland, P., Chowdhury, A. M. R. and RamosJimenez, P. Patterns of vaccination acceptance. Soc. Sci. Med. 1999; 49: 1705-1716.

9. Streefland, P. H., Chowdhury, A. M. and RamosJimenez, P. Quality of vaccination services and social demand for vaccinations in Africa and Asia. Bull. World. Health. Org. 1999; 77: 722- 730.

10. WHO/Regional Office for Africa. Report on the $1^{\text {st }}$ Consultation of the Technical Advisory Group on Measles and Rubella Control in the African Region. Division of Prevention and Control of Communicable Diseases. April 2005 (http://www. afro.who.int/ddc/opd/tfi2005/presentations/background docs $t$ fi/Regional\%20Measles \%20TAG\%20Report\%2 0Apr\%2005.pdf)

11. Berhane, Y., Wall, S., Kebede, O., et al. Establishing an epidemiological field laboratory in rural areas - potentials for public health research and intervention. Ethiop. J. Health Dev. 1999; 13(Special Issue): $1-47$.

12. Ministry of Health. Measles Health Facility Survey in Ethiopia. Addis Ababa. 2002.

13. Ethiopian Demographic and Health Survey (DHS), Central Statistical Authority, Addis Ababa. 2005.

14. Ministry of Health. Measles supplemental immunization household survey in Ambo and Yaya districts, Oromiya Regional State, Ethiopia (survey report). Addis Ababa, November 2003.

15. Ministry of Health. Measles supplemental immunization household survey in Amhara and Tigray regional states, Ethiopia (survey report), Addis Ababa, January 2004.

16. Salama, P., Fitsum, A., Leisel, T. et al. Malnutrition, measles, mortality and the humanitarian response during a famine in Ethiopia. J. Am. Med. Assoc. 2001; 286: 563-571.

17. Talley, L. and Salama, P. Assessing field vaccine efficacy for measles in famine-affected rural Ethiopia. Am. J. Trop. Med. Hyg. 2003; 68: 545-546. 